\title{
Wigner Distribution Analysis and the Riemann Hypothesis
}

\author{
TAKAAKI MUSHA ${ }^{1,2}$ \\ ${ }^{1}$ Advanced Science-Technology Research Organization, Yokohama, JAPAN \\ ${ }^{2}$ Foundation of Physics Research Center (FoPRC), Cosenza, ITALY
}

\begin{abstract}
Wigner distribution is a tool for signal processing to obtain instantaneous spectrum of a signal. From which, another representation of the Euler product can be obtained for Dirichlet series of the Mobius function, which leads to the proof of the Riemann hypothesis.
\end{abstract}

Keywords: Wigner-Ville distribution, Mobius function, Euler product, Riemann Hypothesis

Received: May 6, 2020. Revised: September 18, 2020. Accepted: September 30, 2020. Published: October 15, 2020.

\section{Introduction}

The Wigner-Ville distribution is a transform technique used in both the time and frequency domain for the signal processing theory. The main characteristics of this transform is that it is not limited by the uncertainty relation of time and frequency. It was originally proposed by E.Wigner in the context of quantum mechanics in 1932 [1] and later J.Ville introduced it for signal analysis in 1948[2]. The Wigner-Ville distribution (abbreviated Wigner distribution hereafter) is defined by the combination of the Fourier transform and correlation calculation as $W_{x}(\omega, t)=\int_{-\infty}^{+\infty} x(t+\tau / 2) \bar{x}(t-\tau / 2) e^{-i \omega \tau} d \tau$, where $\bar{x}(t)$ is a conjugate of $x(t)$.
This transformation has the advantage of high resolution of signals compared with the Fourier transform and it is often utilized as a tool to obtain instantaneous spectrum of signals. In this paper, the author tries to prove the Riemann hypothesis by using the Euler products for the Dirichlet series of the Mobius function obtained from the Wigner distribution analysis.

\section{Euler Product of The Dirichlet Series by The}

\section{Wigner Distribution Analysis}

For the Dirichlet series given by

$z(s)=\sum_{n=1}^{\infty} a(n) / n^{s}$, we define the Wigner distribution function shown as

$$
W_{z}(\omega, t)
$$




$$
\begin{aligned}
W_{z}(\omega, t)= & \int_{-\infty}^{+\infty} z(\sigma-i[t+\tau / 2]) \\
& \times \bar{z}(\sigma-i[t-\tau / 2]) e^{-i \omega \tau} d \tau,
\end{aligned}
$$

where $s$ is a complex number given by $s=\sigma+i t$. As $z(s)$ can be rewritten as $z(\sigma-i t)=\sum_{n=1}^{\infty} \frac{a(n) e^{i t \log n}}{n^{\sigma}}$ by real parameters $\sigma$ and $t$, then $W_{z}(\omega, t)$ can be given by

$$
\begin{aligned}
& W_{z}(\omega, t)=\int_{-\infty}^{+\infty} \sum_{k=1}^{\infty} \frac{a(k)}{k^{\sigma}} \exp [i(t+\tau / 2) \log k] \\
& \times \sum_{l=1}^{\infty} \frac{a(l)}{l^{\sigma}} \exp [-i(t-\tau / 2) \log l] e^{-i \omega \tau} d \tau \\
& =\int_{-\infty}^{+\infty} \sum_{k, l}^{\infty} \frac{a(k) a(l)}{(k l)^{\sigma}} \exp [i \log (k / l) t] \exp [i \log (k l) \tau / 2] e^{-i \omega \tau} d \tau
\end{aligned}
$$

Put $n=k l$ and rearranging the equation, we have $W_{z}(\omega, t)$

$=\sum_{k, l}^{\infty} \frac{a(k) a(l)}{n^{\sigma}} \exp [i \log (k / l) t] \int_{-\infty}^{+\infty} \exp (i \log n \cdot \tau / 2) e^{-i \omega \tau} d \tau$ $=2 \pi \sum_{k, l}^{\infty} \frac{a(k) a(l)}{n^{\sigma}} \exp [i \log (k / l) t] \delta\left(\omega-\frac{\log n}{2}\right)$ $=2 \pi \sum_{n=1}^{\infty} \frac{1}{n^{\sigma}} \delta\left(\omega-\frac{\log n}{2}\right) \cdot \sum_{n=k l} a(k) a(l) \exp [i \log (k / l) t]$ where $\delta(\omega)$ is a Dirac delta function.

We let $b(n, t)=\sum_{n=k l} a(k) a(l) \exp [i \log (k / l) t]$, the Wigner distribution function of the Dirichlet series $z(s)$ becomes

$W_{z}(\omega, t)=2 \pi \sum_{n=1}^{\infty} \frac{b(n, t)}{n^{\sigma}} \delta\left(\omega-\frac{\log n}{2}\right)$.

To obtain the Euler product by the Wigner distribution analysis, we have to prove following Lemmas at first.

Lemma.1: Let $\tau=t / 2$ and $s^{\prime}=\sigma+i \tau$, we have $z(s)=z(\sigma)^{-1} \sum_{n=1}^{\infty} \frac{b(n, \tau)}{n^{s^{\prime}}}$.

Proof; We utilize the property of the Wigner distribution shown as [3] $\frac{1}{2 \pi} \int_{-\infty}^{+\infty} W_{f}(\omega, t / 2) e^{i \omega t} d \omega=\bar{f}(0) f(t)$.

As the left side integral of this equation yields

$\frac{1}{2 \pi} \int_{-\infty}^{+\infty} W_{z}(\omega, t / 2) e^{i \omega t} d \omega=\sum_{n=1}^{\infty} \frac{b(n, t / 2)}{n^{\sigma}} \int_{-\infty}^{+\infty} \delta\left(\omega-\frac{\log n}{2}\right) \cdot e^{i \omega t} d \omega$

$=\sum_{n=1}^{\infty} \frac{b(n, t / 2)}{n^{\sigma}} \exp \left(i \frac{t}{2} \log n\right)$

then we have

$$
z(\sigma-i t)=z(\sigma)^{-1} \sum_{n=1}^{\infty} \frac{b(n, t / 2)}{n^{\sigma}} \exp \left(i \frac{t}{2} \log n\right) .
$$

From the definition of $b(n, t)$, we can see $b(n, t)=b(n,-t)$, then Eq.(1) can be rewritten as

$$
\begin{aligned}
& z(\sigma+i t)=z(\sigma)^{-1} \sum_{n=1}^{\infty} \frac{b(n, t / 2)}{n^{\sigma}} \exp \left(-i \frac{t}{2} \log n\right) \\
& =z(\sigma)^{-1} \sum_{n=1}^{\infty} \frac{b(n, \tau)}{n^{s^{\prime}}}
\end{aligned}
$$

We let $\tau=t / 2$ and $s^{\prime}=\sigma+i \tau$, Lemma. 1 can be obtained.

Lemma.2: Let $a(n)$ be a multiplicative function, then $b(n, t)$ is a multiplicative function satisfying $b(m n, t)=b(m, t) \cdot b(n, t)$.when $(m, n)=1$.

Proof; From the definition of $b(n, t)$, we have $b(n, t)=\sum_{d \mid n} a(d) a(n / d) \exp \left[i t \log \left(d^{2} / n\right)\right]$.

Put $n=n_{1} n_{2}$, and $d=d_{1} d_{2}$, which satisfy $\left(n_{1} n_{2}\right)=1,\left(d_{1} d_{2}\right)=1$, then we can write

$$
\begin{aligned}
& \sum_{d_{1} d_{2} \mid n_{1} n_{2}} a\left(d_{1} d_{2}\right) a\left(n_{1} n_{2} /\left(d_{1} d_{2}\right)\right) \exp \left[i t \log \left(\left(d_{1} d_{2}\right)^{2} /\left(n_{1} n_{2}\right)\right)\right] \\
= & \sum_{d_{1} \mid n_{1}} a\left(d_{1}\right) a\left(n_{1} / d_{1}\right) \exp \left[i t \log \left(d_{1}^{2} / n_{1}\right)\right]
\end{aligned}
$$

.$\times \sum_{d_{2} \mid n_{2}} a\left(d_{2}\right) a\left(n_{2} / d_{2}\right) \exp \left[i t \log \left(d_{2}^{2} / n_{2}\right)\right]$

Hence, it can be seen that $b(n, t)$ is a multiplicative function. 
From which, we can obtain the Euler product of the Dirichlet series as follows.

Theorem.1: The Dirichlet series $z(s)$ consisted of a multiplicative function gives the following Euler product.

$\sum_{n=1}^{\infty} \frac{a(n)}{n^{s}}=z(\sigma)^{-1} \prod_{p}\left(1+\sum_{r=1}^{\infty} \frac{b\left(p^{r}, \tau\right)}{p^{r s^{\prime}}}\right)$

where $s^{\prime}=\sigma+i \tau$.

Proof; If we let $g(n)$ be a multiplicative function, we have

$\sum_{n=1}^{\infty} g(n)=\prod_{p}\left(1+g(p)+g\left(p^{2}\right)+g\left(p^{3}\right)+\cdots\right)$,

if satisfying $\sum_{p, r}\left|g\left(p^{r}\right)\right|<+\infty[4]$.

From Lemma.2, $b(n, \tau)$ is a multiplicative function, thus we have

$$
\sum_{n=1}^{\infty} \frac{b(n, \tau)}{n^{s^{\prime}}}=\prod_{p}\left(1+\sum_{r=1}^{\infty} \frac{b\left(p^{r}, \tau\right)}{p^{r s^{\prime}}}\right)
$$

Then we can obtain Eq.(2) from Lemma.1.

\section{Euler Product of The Dirichlet Series of The}

\section{Mobius Function}

We try to obtain Euler products of the Dirichlet series of the Mobius function shown as follows:

Theorem.2 Let $s=\sigma+i t$ and $s^{\prime}=\sigma+i \tau$, where $\tau=t / 2$, the Dirichlet series of the Mobius function has the Euler product given by

$\sum_{n=1}^{\infty} \frac{\mu(n)}{n^{s}}=\zeta\left(\operatorname{Re}\left[s^{\prime}\right]\right) \prod_{p}\left(1-2 p^{-s^{\prime}} \cos \left(\operatorname{Im}\left[s^{\prime}\right] \log p\right)+p^{-2 s^{\prime}}\right)$

for $\sigma \geq \Omega$ satisfying $\Theta \geq \Omega$, where $\Theta$ is the lower limit that the Dirichlet series of the Mobius function is absolutely convergent $(1 / 2 \leq \Theta \leq 1)[4]$ and $\zeta(s)$ is the Riemann zeta function.
.Proof; From Lemma.2, $b(n, \tau)$ is a multiplicative function because it can be given by $b(n, t)=\sum_{n=k l} a(k) a(l) \exp [i \log (k / l) t]$.

From which, we have $b(p, \tau)=-2 \cos (\tau \log p), b\left(p^{2}, \tau\right)=1$ and $b\left(p^{r}, \tau\right)=0 \quad(r \geq 3), \quad$ then we obtain $1+\sum_{r=1}^{\infty} \frac{b\left(p^{r}, \tau\right)}{p^{r s^{\prime}}}=1-2 p^{-s^{\prime}} \cos (\tau \log p)+p^{-2 s^{\prime}}$.

When we let $m(s)=\sum_{n=1}^{\infty} \frac{\mu(n)}{n^{s}}$, we have

$\prod_{p}\left(1-2 p^{-s^{\prime}} \cos (\tau \log p)+p^{-2 s^{\prime}}\right)=\prod_{p}\left(1-2 p^{-\sigma}+p^{-2 \sigma}\right)$

at $s^{\prime}=\sigma$, then $m(\sigma)^{2}=\prod_{p}\left(1-p^{-\sigma}\right)^{2}$.

From which, we have $m(\sigma)=1 / \zeta(\sigma)$.

Then we have

$\sum_{n=1}^{\infty} \frac{\mu(n)}{n^{s}}=\zeta(\sigma) \prod_{p}\left(1-2 p^{-s^{\prime}} \cos (\tau \log p)+p^{-2 s^{\prime}}\right)$

from Theorem.1. Hence we obtain Eq.(3).

As $b(n, \tau)=\sum_{d \mid n} \mu(d) \mu(n / d) \exp \left[i \tau \log \left(d^{2} / n\right)\right]$

$=\sum_{n=k l} \mu(k) \mu(l) \exp [i \tau \log (k / l)]$

then we have

$$
\begin{aligned}
& \left(\sum_{n=1}^{\infty} \frac{|\mu(n)|}{n^{\sigma}}\right)^{2}=\left(\sum_{k=1}^{\infty} \frac{|\mu(k)|}{k^{\sigma}}\right) \cdot\left(\sum_{l=1}^{\infty} \frac{|\mu(l)|}{l^{\sigma}}\right) \\
& \geq \sum_{n=1}^{\infty} \sum_{n=k l} \frac{|\mu(k)| \cdot|\mu(l)| \cdot|\cos (\tau \log (k / l))|}{(k l)^{\sigma}} \\
& >\sum_{n=1}^{\infty}\left|\sum_{n=k l} \frac{\mu(k) \mu(l) \cos (\tau \log (k / l)) \mid}{(k l)^{\sigma}}\right|=\sum_{n=1}^{\infty} \frac{|b(n, \tau)|}{n^{\sigma}}
\end{aligned}
$$

,because

$\exp [i \tau \log (k / l)]+\exp [i \tau \log (l / k)]=2 \cos (\tau \log (k / l))$

When we let $\Omega$ be the lower limit that $\sum_{n=1}^{\infty} \frac{|b(n, \tau)|}{n^{\sigma}}$

converges, Theorem.2 can be obtained. 


\section{Evaluation of an Infinite Product}

The problem of the Riemann hypothesis is whether the Euler product for the Riemann zeta function is convergent or not for $\sigma>1 / 2$ as pointed out by Kimura, Koyama and Kurokawa in their paper [5]. As the Dirichlet series of the Mobius function can be given by

$$
\begin{aligned}
& \frac{1}{\zeta(s)}=\left(1-\frac{1}{p_{1}^{s}}\right) \cdot\left(1-\frac{1}{p_{2}^{s}}\right) \cdot\left(1-\frac{1}{p_{3}^{s}}\right) \ldots \\
& =1-\lim _{N \rightarrow \infty}\left(\sum_{0<i<N} \frac{1}{p_{i}^{s}}-\sum_{0<i<j<N} \frac{1}{p_{i}^{s} p_{j}^{s}}+\sum_{0<i<j<k<N} \frac{1}{p_{i}^{s} p_{j}^{s} p_{k}^{s}}-\cdots\right)
\end{aligned}
$$

$=\sum_{n=1}^{\infty} \frac{\mu(n)}{n^{s}}[6]$, then we consider the convergence of the infinite product in Eq.(3), shown as follows;

Lemma.3, If $\tau \neq 0$ and $\sigma>0$, we have

$$
1-p^{-2 \sigma} \geq\left|1-2 p^{-s^{\prime}} \cos (\tau \log p)+p^{-2 s^{\prime}}\right| \geq\left(1-p^{-\sigma}\right)^{2}
$$

\section{Proof;}

If $\tau=0$, we have

$$
\left|1-2 p^{-s^{\prime}} \cos (\tau \log p)+p^{-2 s^{\prime}}\right|=\left(1-p^{-\sigma}\right)^{2} .
$$

From which, we have

$$
\sum_{n=1}^{\infty} \frac{\mu(n)}{n}=\prod_{p}\left(1-p^{-1}\right)=0, \text { which is equivalent }
$$

to the prime number theorem [7].

For the case $\tau \neq 0$,

$$
\begin{aligned}
& \left|1-2 p^{-s^{\prime}} \cos (\tau \log p)+p^{-2 s^{\prime}}\right| \\
& =\left[\left(1-2 p^{-\sigma} \cos ^{2}(\tau \log p)+p^{-2 \sigma} \cos (2 \tau \log p)\right)^{2}\right. \\
& \left.+\left(2 p^{-\sigma} \sin (\tau \log p) \cos (\tau \log p)-p^{-2 \sigma} \sin (2 \tau \log p)\right)^{2}\right]^{1 / 2} \\
& =\left[1+p^{-4 \sigma}-2 p^{-2 \sigma}-\left(4 p^{-\sigma}-8 p^{-2 \sigma}+4 p^{-3 \sigma}\right) \cos ^{2}(\tau \log p)\right]^{1 / 2} \\
& =\left[1+p^{-4 \sigma}-2 p^{-2 \sigma}-\left(4 p^{-\sigma}-8 p^{-2 \sigma}+4 p^{-3 \sigma}\right) \cos ^{2}(\tau \log p)\right]^{1 / 2}
\end{aligned}
$$

At $\cos ^{2}(\tau \log p)=0$, it has a maximum value

$$
\left(1+p^{-4 \sigma}-2 p^{-2^{\sigma}}\right)^{1 / 2}=1-p^{-2 \sigma} \quad, \quad \text { and } \quad \text { at }
$$

$\cos ^{2}(\tau \log p)=1$, it has a minimum value

$\left(1-p^{-\sigma}\right)^{2}$, because

$$
1+p^{-4 \sigma}-4 p^{-\sigma}+6 p^{-2 \sigma}-4 p^{-3 \sigma}=\left(1-p^{-\sigma}\right)^{4}
$$

Then we have

$1-p^{-2 \sigma} \geq\left|1-2 p^{-s^{\prime}} \cos (\tau \log p)+p^{-2 s^{\prime}}\right| \geq\left(1-p^{-\sigma}\right)^{2}$

\section{Lemma.4}

$\prod_{p}\left(1 \pm p^{-2 \sigma}\right)$ is convergent for $\sigma>1 / 2$.

\section{Proof;}

$\prod_{p} \log \pm \overbrace{}^{-2 \sigma}=) \sum_{p} \log \notin p^{-2 \sigma})$.

From $\log (1+x)=x-\frac{x^{2}}{2}+\frac{x^{3}}{3}-\cdots$,

$\log \left(1 \pm p^{-2 \sigma}\right)= \pm p^{-2 \sigma} \mp \frac{p^{-4 \sigma}}{2} \pm \frac{p^{-6 \sigma}}{3} \mp \cdots$,

then

$$
\begin{aligned}
& \log \prod_{p}\left(1 \pm p^{-2 \sigma}\right) \\
= & \pm \sum_{p} p^{-2 \varsigma} \mp \frac{1}{2} \sum_{p} p^{-4 \sigma} \pm \frac{1}{3} \sum_{p} p^{-6 \sigma} \mp \ldots
\end{aligned}
$$

which is an alternating series.

$$
\begin{aligned}
& \zeta(k)=\sum_{n=1}^{\infty} \frac{1}{n^{k}}>\sum_{p} p^{-k} \text { and } \\
& \frac{1}{n} \sum_{p} p^{-2 n \sigma}>\frac{1}{n+1} \sum_{p} p^{-2(n+1) \sigma}, \text { then } \\
& \prod_{p}\left(1 \pm p^{-2 \sigma}\right) \quad \text { is } \quad \text { convergent for } \sigma>1 / 2
\end{aligned}
$$

$$
\text { because } \lim _{n \rightarrow \infty} \frac{1}{n} \sum_{p} p^{-2 n \sigma}=0 .
$$

\section{Lemma.5}

$\left|\prod_{p}\left(1-2 p^{-s^{\prime}} \cos (\tau \log p)+p^{-2 s^{\prime}}\right)\right|$ is convergent for $\sigma>1 / 2$.

Proof; 
From Lemma.3 and 4, the convergence area of an infinite product

$$
\left|\prod_{p}\left(1-2 p^{-s^{\prime}} \cos (\tau \log p)+p^{-2 s^{\prime}}\right)\right|
$$

is bounded for $1 \geq \sigma>1 / 2$.

According to the Bolzano-Weierstrass theorem, the infinite product (4) has an accumulation point, because its convergence area is bounded..

When we let $P_{m}=\left|1-2 p_{m}^{-s^{\prime}} \cos \left(\tau \log p_{m}\right)+p_{m}^{-2 s^{\prime}}\right|$ , we have

$$
\begin{aligned}
\left|P_{m}-P_{n}\right| & \leq\left|1-p_{m}^{-2 \sigma}-\left(1-p_{n}^{-\sigma}\right)^{2}\right| \\
& =\left|p_{m}^{-2 \sigma}+p_{n}^{-2 \sigma}-2 p_{n}^{-\sigma}\right|,
\end{aligned}
$$

from Lemma 3, where $p_{m}$ and $p_{n}$ are the m-th prime and n-th prime, respectively.

As Eq.(5) converges zero when $m, n \rightarrow \infty$ for $\sigma>1 / 2$, then this is a Cauchy sequence, the terms of which are getting closer together in a way that the sequence ought to have a limit.

If $\left|P_{m}-P_{n}\right| \rightarrow 0$, then $P_{m} / P_{n}$ approaches unity, the absolute value of the infinite product, $\left|\prod_{p}\left(1-2 p^{-s^{\prime}} \cos (\tau \log p)+p^{-2 s^{\prime}}\right)\right|$, converges to a certain value for $\sigma>1 / 2$.

As the maximum absolute value of Eq.(3) is less than

$$
\begin{aligned}
& \zeta(\sigma) \prod_{p}\left(1-p^{-2 \sigma}\right)=\prod_{p} \frac{\left(1+p^{-\sigma}\right)\left(1-p^{-\sigma}\right)}{1-p^{-\sigma}} \\
& =\prod_{p}\left(1+p^{-\sigma}\right)
\end{aligned}
$$

from Lemma.3, then we use $1+p^{-2 \sigma}$ for the evaluation of the infinite product given by Eq.(4) instead of $1-p^{-2 \sigma}$, which satisfies $1+p^{-2 \sigma}>1-p^{-2 \sigma} \quad$ for $\quad \sigma \geq 0$, because $\prod_{p}\left(1+p^{-\sigma}\right)$ is not convergent for $\sigma \leq 1$.

Then the following theorem can be obtained.

\section{Theorem.3}

$\frac{\zeta(2 \sigma)}{\zeta(4 \sigma)} \geq\left|\prod_{p}\left(1-2 p^{-s^{\prime}} \cos (\tau \log p)+p^{-2 s^{\prime}}\right)\right| \quad$ for $\sigma>1 / 2$.

Proof; As $1+p^{-2 \sigma}>1-p^{-2 \sigma}$ for $\sigma \geq 0$, then we have

$$
\prod_{p}\left(1+p^{-2 \sigma}\right) \geq\left|\prod_{p}\left(1-2 p^{-s^{\prime}} \cos (\tau \log p)+p^{-2 s^{\prime}}\right)\right|
$$
for $\sigma>1 / 2$ from Lemma.4 and 5 .

From $\prod_{p}\left(1+p^{-2 \sigma}\right)=\frac{\zeta(2 \sigma)}{\zeta(4 \sigma)}[8]$, Theorem.3 is obtained.

According to this theorem, it follows that $\sum_{n=1}^{\infty} \frac{\mu(n)}{n^{s}}$ is convergent for $\sigma>1 / 2$ from Theorem.2, by using the formula, $\zeta(\sigma)=\varphi(\sigma) /\left(1-2^{1-\sigma}\right)$, where $\varphi(s)$ is the Dirichlet eta function, then we have the following result.

Corollary: $|\zeta(s)|$ has no zero points for $1 / 2<\sigma<1$.

Proof; From Theorem.3, we have $\frac{\zeta(2 \sigma)}{\zeta(4 \sigma)} \geq\left|\prod_{p}\left(1-2 p^{-2 s^{\prime}} \cos (\tau \log p)+p^{-2 s^{\prime}}\right)\right|$ where $\sigma>1 / 2$ and $\operatorname{Im}\left[s^{\prime}\right] \neq 0$

As $\sum_{n=1}^{\infty} \frac{\mu(n)}{n^{s}}=\frac{1}{\zeta(s)}$, we have 


$$
|\zeta(s)| \geq|\zeta(\sigma)|^{-1} \frac{\zeta(4 \sigma)}{\zeta(2 \sigma)}=\frac{\left|1-2^{1-\sigma}\right|}{|\varphi(\sigma)|} \frac{\zeta(4 \sigma)}{\zeta(2 \sigma)}
$$

$(\operatorname{Im}[s] \neq 0)$ from Theorem.2, which satisfies $|\zeta(1 / 2+i t)| \geq 0$, by using the Dirichlet eta function $\varphi(s)$, which is given by

$$
\zeta(\sigma)=\varphi(\sigma) /\left(1-2^{1-\sigma}\right) \text { for } \sigma<1 .
$$

Then, the plot of $|\zeta(\sigma)|^{-1} \zeta(4 \sigma) / \zeta(2 \sigma)$ is shown as follows;

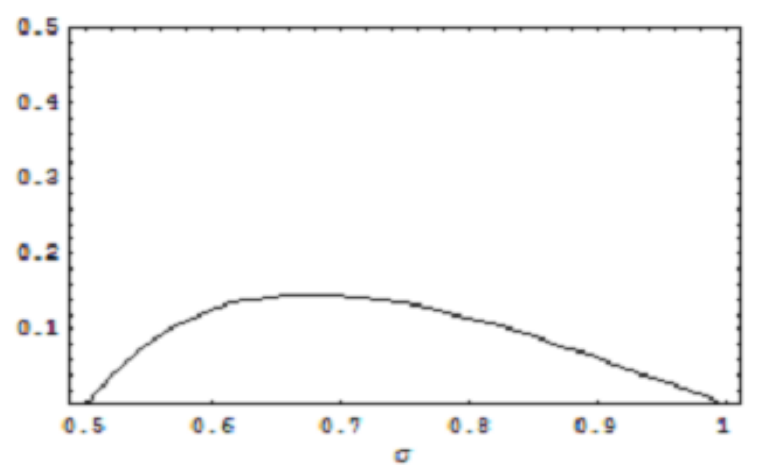

Figure.1 Plot of $|\zeta(\sigma)|^{-1} \zeta(4 \sigma) / \zeta(2 \sigma)$

As shown in Fig.1, we have $|\zeta(s)|>0$ for $1 / 2<\sigma<1$ and the Riemann zeta function has no zeros between $1 / 2$ and 1 for $\operatorname{Im}[s] \neq 0$. The Riemann zeta function has no zeros on the real axis $(\tau=0)$ for $0<\sigma<1[9]$, then $|\zeta(s)|$ does not become zero for $1 / 2<\sigma<1$.

\section{Conclusion}

By the Wigner-Ville distribution analysis, which is a tool developed for analyzing instantaneous spectrum of a signal, it can be shown that the Riemann zeta function has no zero points for $1 / 2<\operatorname{Re}[s]<1$, which shows that all nontrivial Riemann zeta function zeros lie on the critical line from the functional equation for the Riemann zeta function.

\section{References}

[1]. E.Wigner, On the Quantum Correlations for Thermodynamic Equilibrium, Phys.Rev., Vol.40 (1932), 749-759.

[2]. J.Ville, Theory et Application de la Notion de Signal Analytique, Cables et Transmissions, Vol.20A (1948), 61-77.

[3]. T.A.C.M., Claasen and W.F.G. Mecklenbrauker, The Wigner Distribution- A Tool for Time-Frequency Signal Analysis (PART.I), Philips J.Res.35 (1980), 217-250.

[4]. D.P.Paran, Exercices de theorie des nombres, Springer-Verlag Tokyo,1987.

[5]. T.Kimura, S.Koyama, N.Kurokawa, Euler Product beyond the Boundary, Letters in Mathematical Physics, 104 (2014), 1-19.

[6]. J.Derbyshire, Prime Obsession: Bemhart Riemann and the Greatest Unsolved Problem in Mathematics, Penguin Books, New York, 2004.

[7]. P.Ribenboim, The Little Book of Big Primes, Springer-Verlag, New York, 1991.

[8]. E.C.Titchmarsh, The Theory of the Riemann Zeta-functions, Oxford University Press, Oxford,1999.

[9]. J.Stopple, A Primer of Analytic Number Theory, Cambridge University Press, 2003.

\section{Creative Commons Attribution License 4.0 (Attribution 4.0 International, CC BY 4.0)}

This article is published under the terms of the Creative Commons Attribution License 4.0 https://creativecommons.org/licenses/by/4.0/deed.en_US 